\title{
Double bronchodilation in chronic obstructive pulmonary disease: a crude analysis from a systematic review
}

This article was published in the following Dove Press journal:

International Journal of COPD

23 June 2017

Number of times this article has been viewed

Jose Luis Lopez-Campos ${ }^{1,2}$

Carmen Calero-Acuña ${ }^{1,2}$

Eduardo Márquez-Martín'

Esther Quintana Gallego, ${ }^{1,2}$

Laura Carrasco-Hernández'

Maria Abad Arranz'

Francisco Ortega Ruiz ${ }^{1,2}$

'Virgen del Rocio University Hospital, Biomedicine Institute of Seville (IBiS), Hospital Universitario Virgen del Rocío, Universidad de Sevilla, Sevilla, Spain; ${ }^{2}$ Centro de Investigación Biomédica en Red de Enfermedades Respiratorias (CIBERES), Instituto de Salud Carlos III, Madrid, Spain
Correspondence: Jose Luis Lopez-Campos

Instituto de Biomedicina de Sevilla (IBiS), Hospital Universitario Virgen del Rocío, Avenida Manuel Siurot, s/n. 41013 Seville, Spain

Tel/fax+349550I 3167

Email lopezcampos@separ.es
Objective: The combination of a long-acting muscarinic antagonist (LAMA) and a long-acting $\beta_{2}$-agonist (LABA) in a single inhaler is a viable treatment option for patients with chronic obstructive pulmonary disease (COPD). Here, we systematically review the current knowledge on double bronchodilation for the treatment of COPD, with a specific focus on its efficacy versus placebo and/or monotherapy bronchodilation.

Methods: A systematic review of clinical trials investigating LABA/LAMA combination therapies was conducted. Articles were retrieved from PubMed, Embase, and Scopus on June 26, 2016. We specifically selected clinical trials with a randomized controlled or crossover design published in any scientific journal showing the following characteristics: 1) comparison of different LABA/LAMA combinations in a single inhaler for patients with COPD, 2) dose approved in Europe, and 3) focus on efficacy (versus placebo and/or bronchodilator monotherapy) in terms of lung function, respiratory symptoms, or exacerbations.

Results: We analyzed 26 clinical trials conducted on 24,338 patients. All LABA/LAMA combinations were consistently able to improve lung function compared with both placebo and bronchodilator monotherapy. Improvements in symptoms were also consistent versus placebo, showing some lack of correlation for some clinical end points and combinations versus monotherapy bronchodilation. Albeit being an exploratory end point, exacerbations showed an improvement with LABA/LAMA combinations over placebo in some trials; however, scarce information was available in comparison with bronchodilator monotherapy in most studies.

Conclusion: Our data show consistent improvements for LABA/LAMA combinations, albeit with some variability (depending on the clinical end point, the specific combination, and the comparison group). Clinicians should be aware that these are average differences. All treatments should be tailored at the individual level to optimize clinical outcomes.

Keywords: COPD, bronchodilators, efficacy, systematic review

\section{Introduction}

Different combinations of a long-acting muscarinic antagonist (LAMA) and a longacting $\beta_{2}$-agonist (LABA) in a single inhaler are gaining popularity for the treatment of chronic obstructive pulmonary disease (COPD). ${ }^{1-3}$ Owing to their promising pharmacological efficacy ${ }^{4}$ and safety, ${ }^{5}$ LABA/LAMA fixed-dose combination (FDC) therapies - also known as double bronchodilation $-{ }^{6}$ are increasingly considered as a viable therapeutic option for patients with COPD.

Four commercial LABA/LAMA FDCs are currently available in Europe. They include the indacaterol/glycopyrronium (IND/GLY), umeclidinium/vilanterol (UMEC/VIL), aclidinium/formoterol (ACLI/FOR), and tiotropium/olodaterol 
(TIO/OLO) combinations. Each combination had undergone a specific development program and clinical trial testing to assess its efficacy and safety for patients with COPD. Unfortunately, no direct head-to-head comparison of the available combinations has been performed yet, the only exception being a clinical trial assessing tiotropium/indacaterol versus UMEC/VIL. ${ }^{7}$ The results of this study indicated similar improvements in lung function and patient-reported outcomes over a 12-week period, with comparable tolerability and safety profiles. ${ }^{7}$

With direct comparison studies being lacking, indirect comparisons may be useful to guide clinical decision making. To date, at least 4 meta-analyses have assessed the comparative efficacy and safety of different double combinations in patients with COPD. ${ }^{8-11}$ Moreover, 1 review found small mean improvements in health-related quality of life and forced expiratory volume in the first second of expiration $\left(\mathrm{FEV}_{1}\right)$ for patients who received a combination of tiotropium and LABA compared with either agent alone. ${ }^{12}$ Taken together, these data indicate that 1) all LAMA/LABA FDCs are characterized by similar efficacy and safety ${ }^{11}$ and 2) they performed better than a LAMA or a LABA alone (regardless of the drugs used). ${ }^{10}$

However, these analyses are methodologically limited by the approach used for meta-analyses, ${ }^{13}$ mainly consisting in the grouping of different combinations into a unique category ${ }^{9}$ and/or the collapsing of different monotherapy components into a single group. ${ }^{10}$ Unfortunately, this approach may overlook the specific differences in terms of efficacy of different LABA/LAMA FDCs. In this context, we reasoned that a clear, comprehensive, and useful summary of clinical trial data for the four LABA/LAMA FDCs available in Europe may be clinically useful. Specifically, this knowledge would provide clinicians with useful information on the expected outcomes of double bronchodilation in COPD. Here, we systematically review the current knowledge on double bronchodilation for the treatment of COPD, with a specific focus on its efficacy versus placebo and/or bronchodilator monotherapy. Although direct comparisons were not feasible, an assessment of average improvements of different efficacy end points helps clinicians to forecast the expected average benefits, ultimately informing clinical decision making.

\section{Methods}

A systematic review of clinical trials investigating LABA/ LAMA combination therapies was conducted. Articles were retrieved from PubMed, Embase, and Scopus on June 26, 2016.
We performed a separate search for each of the available combinations using the following queries: "QVA149[title] OR (indacaterol[title] AND glycopyrronium[title])" for the IND/GLY combination; "umeclidinium[title] AND vilanterol [title]" for the UMEC/VIL combination; "aclidinium[title] AND formoterol[title]" for the ACLI/FOR combination; and "tiotropium[title] AND olodaterol[title]" for the TIO/ OLO combination. Results were filtered using the following criteria: 1) "clinical trial" in PubMed; 2) "randomized controlled trial" and "crossover procedure" (as study type) in Embase; and 3) "article" (as document type) in Scopus. We retrieved all the abstracts and selected the clinical trials with the following characteristics: 1) randomized controlled or crossover design published in any scientific journal and conducted in patients with COPD; 2) no language restrictions; 3 ) comparison of each LABA/LAMA FDC in a single inhaler; 4) dose approved in Europe; and 5) availability of efficacy data in terms of lung function, respiratory symptoms, or exacerbations versus placebo, tiotropium, or monotherapy bronchodilation (using either LABA or LAMA). Because some articles contained information derived from 2 different trials, both the number of articles and trials were counted. Studies comparing double bronchodilation therapy with an inhaled corticosteroid and LABA combinations or investigations based on post hoc analyses of patient subgroups included in previous trials were excluded. We also excluded the following trials: 1) studies available in a congress abstract form with an associated full-length article, 2) studies evaluating doses different from those currently approved in Europe, 3) studies without a comparison with either placebo or bronchodilator monotherapy, 4) studies evaluating two bronchodilators not combined in the same inhaler, 5) studies providing no efficacy data on lung function, symptoms, or exacerbations, 6) studies that were not original clinical research, and 7) studies conducted in patients other than COPD. After identification of the articles of interest and in an effort to control for potential publication bias, we also contacted the Spanish national representatives of the four companies commercializing the four combinations in Spain. Our aim was to search for any new trial available in a poster form presented at international congresses. Posters were evaluated with the same approach used for original articles; specifically, posters that met the inclusion criteria were incorporated in the analysis (even when the data were not available in a full-length article format). We also reviewed the poster versions of the selected articles to verify the potential presence of any additional information not provided in the corresponding full-length article. 
Upon selection of all studies (ie, both articles and posters), the complete content was reviewed, and information on lung function, symptoms, and exacerbations was retrieved. All the efficacy data were culled from the last visit (ie, at the end of each trial) in the intention-to-treat population. Lung function parameters of interest included $\mathrm{FEV}_{1} 5$ minutes post morning dose (as a measure of the rapid onset of action), peak FEV (defined as the highest $\mathrm{FEV}_{1}$ after morning dose), trough $\mathrm{FEV}_{1}$ (morning pre-dose), and $\mathrm{FEV}_{1}$ area under the curve from 0 to 24 hours post morning dose $\left(\mathrm{FEV}_{1} \mathrm{AUC}_{0-24}\right)$.

The following symptoms were recorded: 1) dyspnea measured by the transitional dyspnea index (TDI); we specifically focused on the mean improvements on this scale and the percentage of patients who showed an improvement of at least 1 TDI point (which is considered the minimum clinically important difference [MCID]), expressed as percentage or odds ratio; 2) health-related quality of life as measured by the St George's Respiratory Questionnaire (SGRQ); we specifically focused on the mean improvements on this scale and the percentage of patients who showed an improvement of at least 4 points in the questionnaire (which is considered the MCID), expressed as percentage or odds ratio; 3 ) rescue medications, as measured in puffs per day (over a 24-hour period); and 4) exercise capacity, as measured by either the endurance time on an exercise test or the endurance shuttle walking test; we specifically focused on the increase during endurance tests (expressed in seconds) as well as on the percentage of increase. We also aimed to include an assessment of daily activities; unfortunately, only the BLAZE ${ }^{14}$ and MOVE ${ }^{15}$ trials focusing on the IND/GLY combination reported such data.

Although exacerbations were not an ad hoc end point in most trials, available information on exacerbations was examined; specifically, we collected both the number of exacerbations occurring during the course of the studies (expressed as annualized rate ratios) and the time to the first exacerbation (expressed as hazard ratios). The extent to which exacerbations were reduced was assessed for all exacerbations and moderate-to-severe exacerbations separately.

All the collected efficacy data were summarized in an Excel spreadsheet, which was shared and checked for accuracy with the Spanish Medical Departments of the four pharmaceutical companies developing the four FDCs. The mean values at the end of the trial were collected for each end point and presented in tables. We constructed one table for each comparator (ie, placebo, own LAMA, tiotropium, and own LABA). The maximum and minimum significant mean improvements observed in different trials were presented for all end points in each table. If no significant differences were evident in a trial, this was noted as the minimum mean improvement. If significant improvements were absent in all the available trials, this was noted as not significant. Because access to raw patient-based data was unavailable, we did not draw any inference on the direct comparison of results; similarly, we were unable to analyze confounders. Direct comparisons were not the specific focus of our study. We rather aimed to provide a general summary of the crude average values of the four different double bronchodilator FDCs, with the ultimate goal of facilitating their clinical evaluation.

\section{Results}

Included and excluded articles (as well as the reasons motivating the exclusion) are shown in Figure 1. Concerning the IND/GLY combination, two posters reporting the results of two clinical trials from the IGNITE initiative (RADIATE and ARISE) were considered as unpublished to date. However, the ARISE poster was excluded because quantitative data on the differences between the treatment arms were not provided. With regard to the ACLI/FOR combination, a poster presenting an extension of the AUGMENT trial ${ }^{16}$ was included. Finally, 2 posters focusing on the TIO/OLO combination in the MORACTO and TORRACTO trials (from the TOVITO initiative) were included; they provided data on the impact of combination therapy on exercise capacity.

The final number of articles and posters included in the analysis was 7 for the IND/GLY combination, 6 for the UMEC/VIL combination, 5 for the ACLI/FOR combination, and 5 for the TIO/OLO combination; there were 7, 8, 3, and 8 trials for each combination, respectively (Figure 1). The available clinical trials and their methodology are summarized in Table S1. The number of patients who were randomized was 6,449 for the IND/GLY combination, 4,011 for the ACLI/FOR combination, 5,886 for the UMEC/VIL combination, and 7,992 for the TIO/OLO combination. There were some differences in terms of patient characteristics across the trials under scrutiny (Table S2). Patients treated with the IND/GLY and ACLI/FOR combinations were similar with regard to lung function impairment and generally included moderate-to-severe cases; they also showed a considerable post-bronchodilator reversibility. Notably, patients treated with the UMEC/VIL combination had more severe disease and showed a lower reversibility. Patients treated with the TIO/OLO combination presented with moderate-to-severe disease and a mean reversibility close to $200 \mathrm{~mL}$.

Efficacy outcomes for each double combination therapy versus placebo are summarized in Table 1. Lung function 


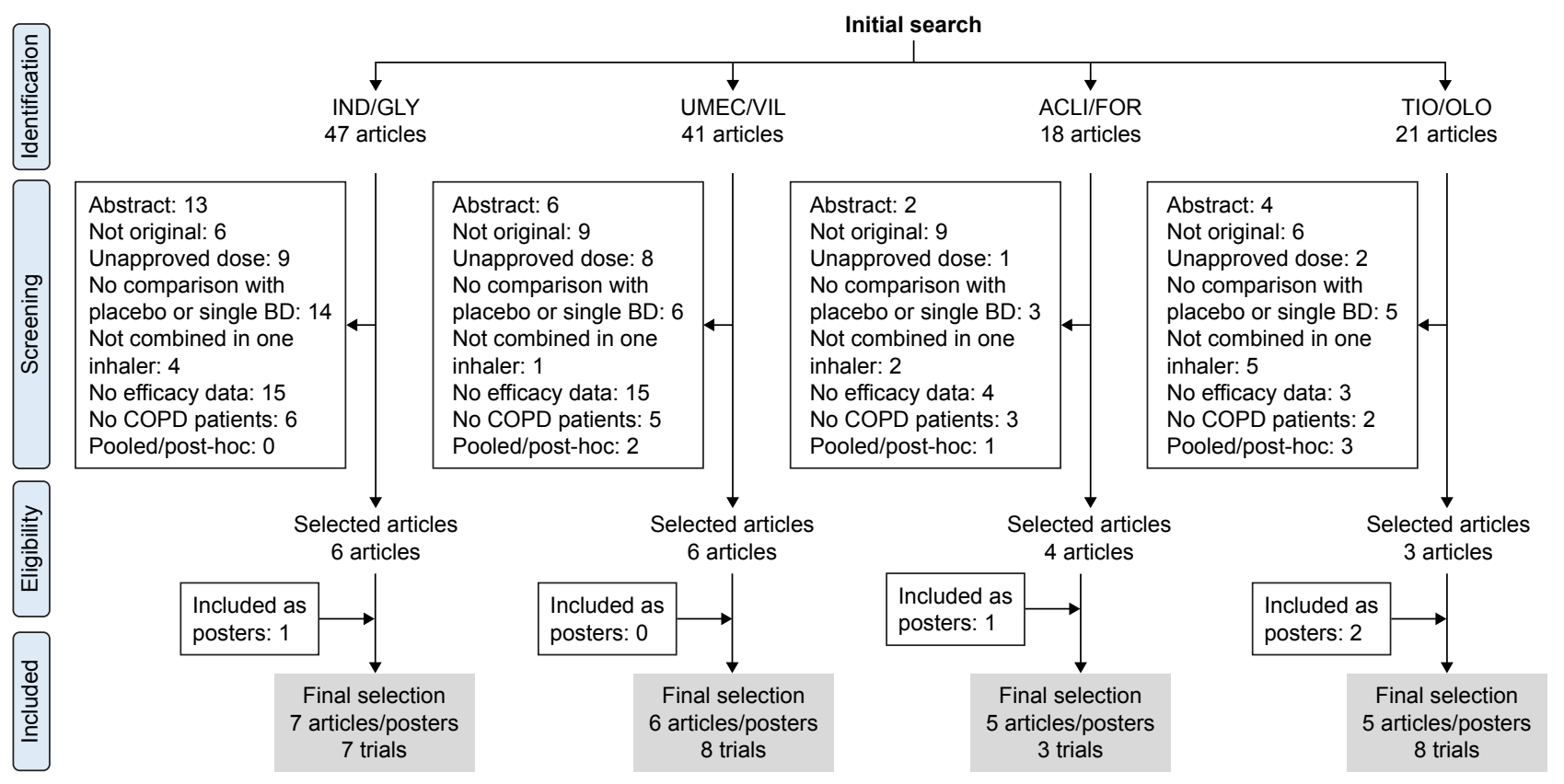

Figure I Flow chart of included-excluded studies.

Abbreviations: ACLI/FOR, aclidinium/formoterol; IND/GLY, indacaterol/glycopyrronium; TIO/OLO, tiotropium/olodaterol; UMEC/VIL, umeclidinium/vilanterol.

showed an improvement in all of the functional parameters under scrutiny. Improvements in dyspnea overcame the MCID for TDI in the majority of studies, with an increase in the number of patients reaching such MCID versus placebo.
Although all combinations improved SGRQ, the IND/GLY combination did not increase the number of patients reaching the MCID for SGRQ in the single study that assessed this end point versus placebo. ${ }^{17}$ Most endurance tests conducted on

Table I Efficacy outcomes for double combinations versus placebo by the end of trial ${ }^{\mathrm{a}}$

\begin{tabular}{|c|c|c|c|c|}
\hline Variables & IND/GLY & UMEC/VIL & ACLI/FOR & TIO/OLO \\
\hline \multicolumn{5}{|l|}{ Lung function } \\
\hline $\mathrm{FEV}, 5$ minutes post morning dose $(\mathrm{mL})$ & 290 & $N A^{b}$ & $108-128$ & $N A^{b}$ \\
\hline Peak FEV, $(\mathrm{mL})$ & 330 & 224 & $298-334$ & 339 \\
\hline Trough FEV $(\mathrm{mL})$ & $189-219$ & $122-243$ & $130-152$ & $162-207$ \\
\hline $\mathrm{FEV}_{1} \mathrm{AUC}_{0-24}(\mathrm{~mL})$ & 320 & $N A^{b}$ & $N A^{b}$ & 280 \\
\hline \multicolumn{5}{|l|}{ Symptoms } \\
\hline Dyspnea (TDI) & $1.09-1.37$ & $0.7-1.2$ & I.3-I.44 & $1.20-2.05$ \\
\hline TDI increase $\geq$ I point $(\%)$ & $10.6-17.8$ & 8 & $16.2-21.6$ & $N^{b}$ \\
\hline TDI increase $\geq I$ point $(O R)$ & 2.78 & $1.3-2$ & $2.54-2.8$ & $N A^{b}$ \\
\hline HRQL (SGRQ) & -3.01 to -4.7 & -2.02 to -5.5 I & NS to -4.36 & -4.56 to -4.89 \\
\hline SGRQ increase $\geq 4$ points (\%) & NSc & $10-11$ & 19.5 & $19.2-21.9$ \\
\hline SGRQ increase $\geq 4$ points (OR) & NS & $1.5-2$ & 2.3 & $2.2-2.5$ \\
\hline Rescue medication (puffs/day) & -0.73 to -1.43 & -0.6 to -1.2 & -0.66 to -0.91 & $N A^{b}$ \\
\hline Endurance test (seconds) & 60 & NS to 69.4 & $N A^{b}$ & $54.9-78.6$ \\
\hline Endurance SWT (seconds) & $N A^{b}$ & $N A^{b}$ & $N A^{b}$ & NSc \\
\hline \multicolumn{5}{|l|}{ Exacerbations } \\
\hline Number of all exacerbations (RR) & $\mathrm{NA}^{\mathrm{b}}$ & $\mathrm{NA}^{\mathrm{b}}$ & NS to 0.76 & $\mathrm{NA}^{\mathrm{b}}$ \\
\hline Time to first exacerbation, all (HR) & 0.7 & $0.5-0.6$ & 0.72 & $N A^{b}$ \\
\hline Number of moderate-to-severe exacerbations (RR) & $N A^{b}$ & $N A^{b}$ & NS to 0.71 & $N A^{b}$ \\
\hline Time to first moderate-to-severe exacerbation (HR) & 0.7 & $N A^{b}$ & 0.70 & $N A^{b}$ \\
\hline
\end{tabular}

Notes: ${ }^{\mathrm{D}}$ Data expressed as the minimum and maximum average value from all trials analyzed. ${ }^{\circ}$ Outcomes with no information in any of the trials evaluated. 'Outcomes with nonsignificant results in all available trials.

Abbreviations: $\mathrm{ACLI} / \mathrm{FOR}$, aclidinium/formoterol; $\mathrm{AUC}_{0-24}$, area under the curve from 0 to 24 hours post morning dose; $F E V_{1}$, forced expiratory volume in the first second; HR, hazard ratio; HRQL, heath-related quality of life; IND/GLY, indacaterol/glycopyrronium; NA, not available; NS, not significant; OR, odds ratio; RR, rate ratio; SGRQ, St George's Respiratory Questionnaire; SWT, shuttle walking test; TDI, transitional dyspnea index; TIO/OLO, tiotropium/olodaterol; UMEC/VIL, umeclidinium/ vilanterol. 
a cycle-ergometer showed an improvement of $\sim 60$ seconds in FDC-treated patients, the only exception being the ACLI/ FOR combination (for which this end point has not been evaluated). The available information on exacerbations showed a decrease in the time to the first exacerbation for all combinations, with the exception being TIO/OLO (for which this end point has not been evaluated). The decrease in the duration and number of exacerbations for the ACLI/ FOR combination (versus placebo) observed in the pooled analysis of the AUGMENT COPD and ACLIFORM studies ${ }^{18}$ was not replicated in individual trials. ${ }^{16,19}$ Although daily activities were not included in this analysis, only the IND/ GLY combination showed some efficacy with respect to this clinical outcome (with the BLAZE trial showing a 8.8\% increase in the percentage of days in which the patient was able to perform usual daily activities versus placebo); ${ }^{14}$ the same combination produced an increase in activity-related energy expenditure (with a $36.7 \mathrm{kcal}$ increase per day versus placebo in the MOVE study). ${ }^{15}$

Efficacy outcomes when each FDC was compared with its own LAMA are summarized in Table 2. The onset of action was reported for all combinations and showed improvements compared with their own LAMA, the only exception being UMEC/VIL (for which this outcome was not reported until 15 minutes after administration).
Peak $\mathrm{FEV}_{1}$ was improved by all combinations, with a slight decrease being reported by UMEC/VIL. Improvements in trough $\mathrm{FEV}_{1}$ ranged from 22 to $90 \mathrm{~mL}$ for all combinations, the only exception being ACLI/FOR. Changes in $\mathrm{FEV}_{1}$ $\mathrm{AUC}_{0-24}$ were investigated for the IND/GLY and TIO/OLO combinations only. The impact on symptoms was not invariably significant. When reported, the TIO/OLO combination resulted in significant improvements versus its LAMA alone for most of the symptoms. With regard to exacerbations, IND/GLY was the only combination that produced significant improvement over its LAMA alone. When the same analysis was performed by comparing an FDC against tiotropium (Table 3), the efficacy parameters were found to improve slightly. The combination ACLI/FOR did not present any studies evaluating its efficacy against tiotropium. With regard to SGRQ, the SPARK study showed that the IND/GLY combination was significantly superior to both glycopyrronium and tiotropium at all assessment points until week 52. ${ }^{20}$ However, the last visit (conducted at week 64) did not show significant differences in the number of patients achieving the MCID.

Efficacy outcomes when each FDC was compared versus its LABA are summarized in Table 4. None of the FDCs differed significantly from their LABA with regard to their onset of action. In line with the LAMA, the improvements

Table 2 Efficacy outcomes for double combinations versus their LAMA by the end of trial ${ }^{\mathrm{a}}$

\begin{tabular}{|c|c|c|c|c|}
\hline Variables & IND/GLY & UMEC/VIL & ACLI/FOR & TIO/OLO \\
\hline \multicolumn{5}{|l|}{ Lung function } \\
\hline $\mathrm{FEV}, 5$ minutes post morning dose $(\mathrm{mL})$ & 130 & $N^{b}$ & 92 & 79 \\
\hline Peak FEV $(\mathrm{mL})$ & 130 & $67-94$ & $|2|-124$ & 111 \\
\hline Trough FEV $(\mathrm{mL})$ & $70-90$ & $22-52$ & NSc & NS-79 \\
\hline $\mathrm{FEV}_{1} \mathrm{AUC}_{0-24}(\mathrm{~mL})$ & 120 & $N A^{b}$ & $\mathrm{NA}^{\mathrm{b}}$ & $\mathrm{III}$ \\
\hline \multicolumn{5}{|l|}{ Symptoms } \\
\hline Dyspnea (TDI) & $N S^{c}$ & $N S^{c}$ & NS-0.44 & $0.35-0.61$ \\
\hline TDI increase $\geq$ I point (\%) & NSc & $N S^{c}$ & NSc & $N^{b}$ \\
\hline TDI increase $\geq I$ point $(O R)$ & $N A^{b}$ & $N S^{c}$ & $N S^{c}$ & $N A^{b}$ \\
\hline HRQL (SGRQ) & NS to -2.8 & $N S^{c}$ & $N S^{c}$ & -1.23 to -2.49 \\
\hline SGRQ increase $\geq 4$ points ( $\%$ ) & NSc,* & $N A^{b}$ & $N S^{c}$ & $7.9-11.4$ \\
\hline SGRQ increase $\geq 4$ points (OR) & NSc,* & NSc & NSc & $1.53-1.58$ \\
\hline Rescue medication (puffs/day) & -0.66 to -0.81 & -0.6 & -0.36 & -0.55 \\
\hline Endurance test (seconds) & $N A^{b}$ & $N A^{b}$ & $N A^{b}$ & $N S^{c}$ \\
\hline Endurance SWT (seconds) & $N A^{b}$ & $N A^{b}$ & $N A^{b}$ & $N A^{b}$ \\
\hline \multicolumn{5}{|l|}{ Exacerbations } \\
\hline Number of all exacerbations (RR) & 0.85 & $N A^{b}$ & $N S^{c}$ & $\mathrm{NA}^{\mathrm{b}}$ \\
\hline Time to first exacerbation, all (HR) & NS $S^{c}$ & NS $S^{c}$ & $N S^{c}$ & $N A^{b}$ \\
\hline Number of moderate-to-severe exacerbations (RR) & 0.88 & $N A^{b}$ & $N S^{c}$ & $N A^{b}$ \\
\hline Time to first moderate-to-severe exacerbation (HR) & $N A^{b}$ & $N A^{b}$ & $N S^{c}$ & NSc \\
\hline
\end{tabular}

Notes: aData expressed as the minimum and maximum average value from all trials analyzed. 'Outcomes with no information in any of the trials evaluated. ${ }^{\circ}$ Outcomes with nonsignificant results in all available trials. *Significant differences favoring the double bronchodilation until week 52.

Abbreviations: $\mathrm{ACLI} / \mathrm{FOR}$, aclidinium/formoterol; $\mathrm{AUC}_{0-24}$, area under the curve from 0 to 24 hours post morning dose; FEV $\mathrm{V}_{1}$ forced expiratory volume in the first second; HR, hazard ratio; HRQL, heath-related quality of life; IND/GLY, indacaterol/glycopyrronium; LAMA, long-acting muscarinic antagonist; NA, not available; NS, not significant; OR, odds ratio; RR, rate ratio; SGRQ, St George's Respiratory Questionnaire; SWT, shuttle walking test; TDI, transitional dyspnea index; TIO/OLO, tiotropium/ olodaterol; UMEC/VIL, umeclidinium/vilanterol. 
Table 3 Efficacy outcomes for double combinations versus tiotropium by the end of trial ${ }^{a}$

\begin{tabular}{|c|c|c|c|c|}
\hline Variables & IND/GLY & UMEC/VIL & ACLI/FOR & TIO/OLO \\
\hline \multicolumn{5}{|l|}{ Lung function } \\
\hline $\mathrm{FEV}, 5$ minutes post morning dose $(\mathrm{mL})$ & 120 & $N A^{b}$ & $N A^{b}$ & 79 \\
\hline Peak FEV $(\mathrm{mL})$ & 130 & $72-95$ & $N A^{b}$ & $\mathrm{III}$ \\
\hline Trough FEV $(\mathrm{mL})$ & $60-100$ & $60-112$ & $N A^{b}$ & NS to 79 \\
\hline $\mathrm{FEV}_{1} \mathrm{AUC}_{0-24}(\mathrm{~mL})$ & 110 & $N A^{b}$ & $N A^{b}$ & 110 \\
\hline \multicolumn{5}{|l|}{ Symptoms } \\
\hline Dyspnea (TDI) & $0.49-0.51$ & $N S^{c}$ & $N A^{b}$ & $0.35-0.61$ \\
\hline TDI increase $\geq$ I point $(\%)$ & $8.9-17.8$ & $N A^{b}$ & $N A^{b}$ & $N A^{b}$ \\
\hline TDI increase $\geq I$ point $(O R)$ & 1.78 & NS $S^{c}$ & $N A^{b}$ & $N A^{b}$ \\
\hline HRQL (SGRQ) & NS to -3.1 & NS to -2.1 & $N A^{b}$ & -1.23 to -2.49 \\
\hline SGRQ increase $\geq 4$ points (\%) & NS* to 7.3 & $N A^{b}$ & $N A^{b}$ & $7.9-11.4$ \\
\hline SGRQ increase $\geq 4$ points (OR) & $N S^{\mathrm{c}, *}$ & NS to 1.4 & $N A^{b}$ & I.53-I.58 \\
\hline Rescue medication (puffs/day) & -0.45 to -1.08 & -0.5 to -0.7 & $N A^{b}$ & -0.55 \\
\hline Endurance test (seconds) & $N S^{c}$ & $N A^{b}$ & $N A^{b}$ & NSc \\
\hline Endurance SWT (seconds) & $N A^{b}$ & $N A^{b}$ & $N A^{b}$ & $N A^{b}$ \\
\hline \multicolumn{5}{|l|}{ Exacerbations } \\
\hline Number of all exacerbations (RR) & 0.86 & NSc & $N A^{b}$ & $N A^{b}$ \\
\hline Time to first exacerbation, all (HR) & NSc & NS to 0.5 & $N A^{b}$ & $N A^{b}$ \\
\hline Number of moderate-to-severe exacerbations (RR) & $N S^{c}$ & $N A^{b}$ & $N A^{b}$ & $N A^{b}$ \\
\hline Time to first moderate-to-severe exacerbation (HR) & NSc & $N A^{b}$ & $N A^{b}$ & $N S^{c}$ \\
\hline
\end{tabular}

Notes: ${ }^{\mathrm{D}}$ Data expressed as the minimum and maximum average value from all trials analyzed. ${ }^{\circ}$ Outcomes with no information in any of the trials evaluated. 'Outcomes with nonsignificant results in all available trials. *Significant differences favoring the double bronchodilation until week 52 .

Abbreviations: $\mathrm{ACLI} / \mathrm{FOR}$, aclidinium/formoterol; $\mathrm{AUC}_{0-24}$, area under the curve from 0 to 24 hours post morning dose; $\mathrm{FEV}_{1}$, forced expiratory volume in the first second; HR, hazard ratio; HRQL, heath-related quality of life; IND/GLY, indacaterol/glycopyrronium; NA, not available; NS, not significant; OR, odds ratio; RR, rate ratio; SGRQ, St George's Respiratory Questionnaire; SWT, shuttle walking test; TDI, transitional dyspnea index; TIO/OLO, tiotropium/olodaterol; UMEC/VIL, umeclidinium/ vilanterol.

Table 4 Efficacy outcomes for double combinations versus their own LABA by the end of trial ${ }^{a}$

\begin{tabular}{|c|c|c|c|c|}
\hline Variables & IND/GLY & UMEC/VIL & ACLI/FOR & TIO/OLO \\
\hline \multicolumn{5}{|l|}{ Lung function } \\
\hline $\mathrm{FEV}, 5$ minutes post morning dose $(\mathrm{mL})$ & NSc & $N A^{b}$ & $N S^{c}$ & $\mathrm{NS}^{\mathrm{c}}$ \\
\hline Peak FEV, $(\mathrm{mL})$ & 120 & $88-116$ & $116-138$ & 120 \\
\hline Trough FEV $(\mathrm{mL})$ & 70 & $90-95$ & $45-81.5$ & $85-92$ \\
\hline $\mathrm{FEV}_{1} \mathrm{AUC}_{0-24}(\mathrm{~mL})$ & 120 & $N A^{b}$ & $\mathrm{NA}^{\mathrm{b}}$ & 115 \\
\hline \multicolumn{5}{|l|}{ Symptoms } \\
\hline Dyspnea (TDI) & $N S^{c}$ & $\mathrm{NS}^{\mathrm{c}}$ & NS to 0.47 & 0.42 \\
\hline TDI increase $\geq$ I point $(\%)$ & NSc & $\mathrm{NA}^{\mathrm{b}}$ & NSc & $\mathrm{NA}^{\mathrm{b}}$ \\
\hline TDI increase $\geq I$ point $(O R)$ & $N A^{b}$ & NSc & NSc & $N A^{b}$ \\
\hline HRQL (SGRQ) & $N S^{c}$ & NS $S^{c}$ & $N S^{c}$ & -1.693 \\
\hline SGRQ increase $\geq 4$ points (\%) & NSc & $N A^{b}$ & $N S^{c}$ & 12.7 \\
\hline SGRQ increase $\geq 4$ points (OR) & $N A^{b}$ & $\mathrm{NS}^{\mathrm{c}}$ & $N S^{c}$ & $N A^{b}$ \\
\hline Rescue medication (puffs/day) & -0.31 & NS $S^{c}$ & $N S^{c}$ & -0.28 \\
\hline Endurance test (seconds) & $N A^{b}$ & $N A^{b}$ & $N A^{b}$ & NS to 46.6 \\
\hline Endurance SWT (seconds) & $N A^{b}$ & $N A^{b}$ & $N A^{b}$ & $N A^{b}$ \\
\hline \multicolumn{5}{|l|}{ Exacerbations } \\
\hline Number of all exacerbations (RR) & $N A^{b}$ & $\mathrm{NA}^{\mathrm{b}}$ & NSc & $\mathrm{NA}^{\mathrm{b}}$ \\
\hline Time to first exacerbation all (HR) & $N A^{b}$ & NSc & NSc & $N A^{b}$ \\
\hline Number of moderate-to-severe exacerbations (RR) & $N A^{b}$ & $N A^{b}$ & $N S^{c}$ & $N A^{b}$ \\
\hline Time to first moderate-to-severe exacerbation (HR) & $N A^{b}$ & $N A^{b}$ & NSc & 0.83 \\
\hline
\end{tabular}

Notes: ${ }^{\mathrm{D}}$ Data expressed as the minimum and maximum average value from all trials analyzed. ${ }^{\mathrm{b} O u t c o m e s}$ with no information in any of the trials evaluated. 'Outcomes with nonsignificant results in all available trials.

Abbreviations: $A C L I / F O R$, aclidinium/formoterol; $A C_{0-24}$, area under the curve from 0 to 24 hours post morning dose; FEV , forced expiratory volume in the first second; HR, hazard ratio; HRQL, heath-related quality of life; IND/GLY, indacaterol/glycopyrronium; LABA, long-acting $\beta_{2}$-agonist; NA, not available; NS, not significant; OR, odds ratio; RR, rate ratio; SGRQ, St George's Respiratory Questionnaire; SWT, shuttle walking test; TDI, transitional dyspnea index; TIO/OLO, tiotropium/olodaterol; UMEC/VIL, umeclidinium/vilanterol. 
in lung function were not accompanied by a lower burden of symptoms or exacerbations for all cases. When reported, only the TIO/OLO combination produced improvements in symptoms and exacerbations in most trials.

\section{Discussion}

This study is the first systematic review focusing on the efficacy of LABA/LAMA FDC through the use of a crude clinical approach that analyzed efficacy data according to different outcomes and comparators. Our data show consistent improvements for LABA/LAMA combinations, albeit with some variability (depending on the clinical end point, the specific combination, and the comparison group).

Here, we specifically focused on efficacy data expressed in terms of average improvements of different clinical outcomes. However, it should be noted that a comprehensive evaluation of different LABA/LAMA FDCs also requires a careful assessment of safety, costs, and, in the case of respiratory medicine, device features. Although a specific analysis of adverse effects was outside the scope of this study, no major alarming side effects were reported by any of the trials under scrutiny. All the LABA/LAMA FDCs had a similar profile in terms of adverse effects, with COPD worsening being the most common adverse manifestation. Other reported side effects included nasopharyngitis, headache, cough, and upper and lower respiratory tract infections; notably, such safety profile was similar to that observed with both placebo and the monocomponents. ${ }^{16,17,21,22}$ Several cost-effectiveness analyses of all LABA/LAMA FDCs have been published, ${ }^{23-26}$ consistently showing that different combinations may have a similar cost-effectiveness ratio. ${ }^{27}$ Another key point is the assessment of the inhaler use. In this regard, a recent study has shown that the inappropriate handling of the inhaler device is underestimated in the real-world practice and portends an increased risk of COPD exacerbations. ${ }^{28}$

Several key aspects need to be considered when interpreting our results. First, the reviewed articles differ significantly in terms of the modality by which several key data (eg, patient characteristics and efficacy outcomes) are expressed. Although some variables were consistently reported by all the included trials (eg, age, percentage of men, and smoking), other clinical data (eg, exacerbations in the previous year or comorbidities) or efficacy parameters (eg, trough $\mathrm{FEV}_{1}$ or breathlessness measured by the TDI questionnaire) were not. For example, the number of patients reaching the MCID in the TDI or SGRQ can be expressed either as odds ratios or percentages. Such discrepancies must be taken into account when different combinations are examined. Other outcomes (including residual functional capacity, residual volume, $\mathrm{FEV}_{1}$ AUC in the first hours after morning dose, $\mathrm{FEV}_{1}$ post dose, percentage of patients improving 100 or $200 \mathrm{~mL}$, and $12 \%$ in trough $\mathrm{FEV}_{1}$ ) were not consistently reported and were not included in the present analysis. Interestingly, the impact of the different combinations on symptoms over the 24 hours of the day has been most extensively studied for the ACLI/ FOR ${ }^{18}$ combination, followed by IND/GLY. ${ }^{29}$ In contrast, no information is available for the UMEC/VIL and TIO/OLO combinations. Night-time and early morning symptoms have been recorded differently (night-time rescue medication use, clinical information in electronic diaries, percentage of nights with no awakenings, or specific questionnaires) in trials examining the ACLI/FOR and IND/GLY combinations. Hopefully, a consensus should be reached on the minimum amount of information that any clinical trial should include, especially in terms of the description of included patients and the presentation of clinical efficacy. Posters obviously include less information than full-length research articles; consequently, data derived from should be considered with caution.

A second point that merits consideration is that the available characteristics of patients included in trials of different combinations were slightly different. Methodological variance (eg, open-label comparisons to tiotropium, different follow-up periods, and use of primary, secondary, or even exploratory outcomes) was also evident. As a result, a raw direct comparison between studies appears unfeasible and was avoided in the present study. At least four meta-analyses have reviewed the available evidence comparing LABA/ LAMA FDCs. Their results showed 1) no differences between the various double FDCs compared with placebo ${ }^{11}$ and 2) the superiority of the combination approach over monotherapy. ${ }^{9,10}$ Even though differences versus placebo were consistent for different combinations, the crude average values indicated that a better lung function was not invariably accompanied by symptom improvement.

Third, another important aspect of our study lies in the assessment of mean values for different efficacy end points. The mean improvement is a simplified modality to express the overall pharmacological response. We are aware that an assessment of the variability of this response (besides average improvement) would be relevant. In general, real-life clinical response to treatments may differ significantly from that observed in a clinical trial, which employs rigid inclusion and exclusion criteria. Recently, Donohue et $\mathrm{al}^{30}$ have shown that the magnitude of UMEC/VIL lung function effect is largely 
dependent on the response to monotherapy, with quantitatively greater improvements in patients who are responders to both umeclidinium and vilanterol monotherapy compared to those responding to one of the two or none of them. The presentation of data as mean improvements together with the number of patients who reached the MCID results is necessary to acquire a more realistic view of the results.

Another area of debate concerns the MCID. Validated MCIDs are available for many commonly used outcomes in COPD, including the $100 \mathrm{~mL}$ improvement in trough $\mathrm{FEV}_{1}$, improvement of $\geq 1$ unit in the TDI total score, reduction of 4 units in the SGRQ total score, or the increase in $47.5 \mathrm{~m}$ for the incremental shuttle walking test, 45-85 seconds for the endurance shuttle walking test, and 46-105 seconds for constant-load cycling endurance tests. ${ }^{31}$ Although the differences compared with placebo reached the MCID for several clinical end points, the differences versus the monotherapy components did not reach the MCID (when significant) for all FDCs. However, numerous factors in a clinical trial setting (eg, study duration, withdrawal rate, or baseline severity) can ultimately influence the measured treatment effects. In addition, it has been questioned whether the same MCID should be used to compare treatment efficacy versus placebo or active treatments. ${ }^{32}$

Finally, another source of controversy lies in the comparison of double bronchodilation versus monotherapy. Despite consistent improvements in lung function compared with placebo, the differences with the monotherapy components were not invariably significant for all the clinical end points and/or comparisons. Interestingly, the correlation of lung function impairment with clinical end points seems to be poor. ${ }^{33}$ As a consequence, the superior efficacy of LABA/ LAMA combinations versus monotherapies in terms of $\mathrm{FEV}_{1}$ might not be paralleled by similar clinical improvements in terms of symptoms and exacerbations.

The onset of action (as reported by $\mathrm{FEV}_{1}$ improvement 5 minutes after the morning dose) was found to be significantly improved compared with placebo and LAMA, but not with LABA. These observations suggest that the rapidity of action of FDCs should be generally ascribed to the LABA. Owing to the first spirometry being performed 15 minutes after the morning dose, the UMEC/VIL study did not provide data at 5 minutes post morning dose. ${ }^{21}$ However, clinical results on vilanterol indicate a rapid onset of action, ${ }^{34}$ suggesting that the effect should be similar when combined with umeclidinium.

Data on exacerbations should be interpreted with caution. In all of the reviewed clinical trials, exacerbations were secondary or even exploratory end points, with the SPARK study ${ }^{20}$ being the only one having exacerbations as a primary end point. Additionally, many trials had a 6-month follow-up, which implies that the rate had to be annualized. As a consequence, the available studies are underpowered to detect differences. Despite these limitations, certain FDCs were characterized by some differences in the time to the first exacerbation compared with placebo, with ACLI/FOR showing a consistent improvement in exacerbation outcomes versus placebo in the pooled analysis. ${ }^{18}$ Of note, the differences in exacerbations versus monotherapy components were either not available or not significant. Only TIO/OLO showed an improvement in the time to the first moderate-to-severe exacerbation compared with the LABA (but not compared with the LAMA) in the TONADO study although exacerbations were not an end point of the trial. ${ }^{22}$

\section{Conclusion}

This study is a systematic review summarizing the crude data obtained from different clinical trials focusing on the efficacy of LABA/LAMA FDCs in patients with COPD. Our data show consistent improvements for LABA/LAMA combinations, albeit with some variability (depending on the clinical end point, the specific combination, and the comparison group). Clinicians should be aware that these are average differences. All treatments should be tailored at the individual level to optimize clinical outcomes.

\section{Acknowledgment}

The authors are grateful to the Spanish teams of Novartis, AstraZeneca, GlaxoSmithKline, and Boehringer Ingelheim for reviewing the efficacy data summarized in the study.

\section{Author contributions}

JLLC designed the study and performed the initial search and wrote the manuscript; JLLC, CCA, and EMM revised the list of publications and posters. EQG, LCH, and MAA extracted the information from the articles. FOR supervised the final draft of the manuscript. All authors contributed toward data analysis, drafting and critically revising the paper, gave final approval of the version to be published, and agree to be accountable for all aspects of the work.

\section{Disclosure}

JLLC has received honoraria for lecturing, scientific advice, participation in clinical studies, or writing publications from the following sources (listed in alphabetical order): Almirall, AstraZeneca, Bayer, Boehringer Ingelheim, Cantabria 
Pharma, Chiesi, Esteve, Faes, Ferrer, GlaxoSmithKline, Menarini, MSD, Novartis, Pfizer, Rovi, and Takeda, and reports no other conflicts of interest in this work. All of the other authors report no conflicts of interest in this work.

\section{References}

1. Dale PR, Cernecka H, Schmidt M, et al. The pharmacological rationale for combining muscarinic receptor antagonists and beta-adrenoceptor agonists in the treatment of airway and bladder disease. Curr Opin Pharmacol. 2014;16:31-42.

2. Cohen JS, Miles MC, Donohue JF, Ohar JA. Dual therapy strategies for COPD: the scientific rationale for LAMA + LABA. Int J Chron Obstruct Pulmon Dis. 2016;11:785-797.

3. Lopez-Campos JL. M(2)-beta(2) interaction: a basis for combined bronchodilator treatment. Arch Bronconeumol. 2013;49(7):279-281.

4. Tashkin DP, Ferguson GT. Combination bronchodilator therapy in the management of chronic obstructive pulmonary disease. Respir Res. 2013;14:49.

5. Matera MG, Rogliani P, Calzetta L, Cazzola M. Safety considerations with dual bronchodilator therapy in COPD: an update. Drug Safety. 2016;39(6):501-508.

6. Lopez-Campos JL, Abad-Arranz M, Calero-Acuna C. Double or dual bronchodilation: defining the correct term. Arch Bronconeumol. 2015; 51(12):661.

7. Kalberg C, O'Dell D, Galkin D, Newlands A, Fahy WA. Dual bronchodilator therapy with umeclidinium/vilanterol versus tiotropium plus indacaterol in chronic obstructive pulmonary disease: a randomized controlled trial. Drugs R D. 2016;16(2):217-227.

8. Huisman EL, Cockle SM, Ismaila AS, Karabis A, Punekar YS. Comparative efficacy of combination bronchodilator therapies in COPD: a network meta-analysis. Int J Chron Obstruct Pulmon Dis. 2015;10:1863-1881.

9. Oba Y, Sarva ST, Dias S. Efficacy and safety of long-acting betaagonist/long-acting muscarinic antagonist combinations in COPD: a network meta-analysis. Thorax. 2016;71(1):15-25.

10. Calzetta L, Rogliani P, Matera MG, Cazzola M. A systematic review with meta-analysis of dual bronchodilation with LAMA/LABA for the treatment of stable COPD. Chest. 2016;149(5):1181-1196.

11. Schlueter M, Gonzalez-Rojas N, Baldwin M, Groenke L, Voss F, Reason T. Comparative efficacy of fixed-dose combinations of longacting muscarinic antagonists and long-acting beta2-agonists: a systematic review and network meta-analysis. Ther Adv Respir Dis. 2016; 10(2):89-104.

12. Farne HA, Cates CJ. Long-acting beta2-agonist in addition to tiotropium versus either tiotropium or long-acting beta2-agonist alone for chronic obstructive pulmonary disease. Cochrane Database Syst Rev. 2015;(10):CD008989.

13. Menzies D. Systematic reviews and meta-analyses. Int J Tuberc Lung Dis. 2011;15(5):582-593.

14. Mahler DA, Decramer M, D'Urzo A, et al. Dual bronchodilation with QVA149 reduces patient-reported dyspnoea in COPD: the BLAZE study. Eur Respir J. 2014;43(6):1599-1609.

15. Watz H, Mailander C, Baier M, Kirsten A. Effects of indacaterol/ glycopyrronium (QVA149) on lung hyperinflation and physical activity in patients with moderate to severe COPD: a randomised, placebocontrolled, crossover study (The MOVE Study). BMC Pulm Med. 2016; 16(1):95

16. D'Urzo AD, Rennard SI, Kerwin EM, et al. Efficacy and safety of fixed-dose combinations of aclidinium bromide/formoterol fumarate: the 24-week, randomized, placebo-controlled AUGMENT COPD study. Respir Res. 2014;15:123.

17. Bateman ED, Ferguson GT, Barnes N, et al. Dual bronchodilation with QVA149 versus single bronchodilator therapy: the SHINE study. Eur Respir J. 2013;42(6):1484-1494.
18. Bateman ED, Chapman KR, Singh D, et al. Aclidinium bromide and formoterol fumarate as a fixed-dose combination in COPD: pooled analysis of symptoms and exacerbations from two six-month, multicentre, randomised studies (ACLIFORM and AUGMENT). Respir Res. 2015;16:92.

19. Singh D, Jones PW, Bateman ED, et al. Efficacy and safety of aclidinium bromide/formoterol fumarate fixed-dose combinations compared with individual components and placebo in patients with COPD (ACLIFORM-COPD): a multicentre, randomised study. BMC Pulm Med. 2014;14:178.

20. Wedzicha JA, Decramer M, Ficker JH, et al. Analysis of chronic obstructive pulmonary disease exacerbations with the dual bronchodilator QVA149 compared with glycopyrronium and tiotropium (SPARK): a randomised, double-blind, parallel-group study. Lancet Respir Med. 2013;1(3):199-209.

21. Decramer M, Anzueto A, Kerwin E, et al. Efficacy and safety of umeclidinium plus vilanterol versus tiotropium, vilanterol, or umeclidinium monotherapies over 24 weeks in patients with chronic obstructive pulmonary disease: results from two multicentre, blinded, randomised controlled trials. Lancet Respir Med. 2014;2(6):472-486.

22. Buhl R, Maltais F, Abrahams R, et al. Tiotropium and olodaterol fixeddose combination versus mono-components in COPD (GOLD 2-4). Eur Respir J. 2015;45(4):969-979.

23. Wilson MR, Patel JG, Coleman A, McDade CL, Stanford RH, Earnshaw SR. Cost-effectiveness analysis of umeclidinium/vilanterol for the management of patients with moderate to very severe COPD using an economic model. Int J Chron Obstruct Pulmon Dis. 2017;12: 997-1008.

24. van Boven JF, Kocks JW, Postma MJ. Cost-effectiveness and budget impact of the fixed-dose dual bronchodilator combination tiotropiumolodaterol for patients with COPD in the Netherlands. Int J Chron Obstruct Pulmon Dis. 2016;11:2191-2201.

25. Ramos M, Haughney J, Henry N, Lindner L, Lamotte M. Cost versus utility of aclidinium bromide 400 microg plus formoterol fumarate dihydrate 12 microg compared to aclidinium bromide 400 microg alone in the management of moderate-to-severe COPD. Clinicoecon Outcomes Res. 2016;8:445-456.

26. Price D, Keininger D, Costa-Scharplatz M, et al. Cost-effectiveness of the LABA/LAMA dual bronchodilator indacaterol/glycopyrronium in a Swedish healthcare setting. Respir Med. 2014;108(12):1786-1793.

27. Tebboth A, Ternouth A, Gonzalez-Rojas N. UK-specific costeffectiveness of tiotropium + olodaterol fixed-dose combination versus other LAMA + LABA combinations in patients with COPD. Clinicoecon Outcomes Res. 2016;8:667-674.

28. Molimard M, Raherison C, Lignot S, et al. Chronic obstructive pulmonary disease exacerbation and inhaler device handling: real-life assessment of 2935 patients. Eur Respir J. 2017;49(2):1601794.

29. Dahl R, Chapman KR, Rudolf M, et al. Safety and efficacy of dual bronchodilation with QVA149 in COPD patients: the ENLIGHTEN study. Respir Med. 2013;107(10):1558-1567.

30. Donohue JF, Singh D, Munzu C, Kilbride S, Church A. Magnitude of umeclidinium/vilanterol lung function effect depends on monotherapy responses: results from two randomised controlled trials. Respir Med. 2016;112:65-74.

31. Jones PW, Beeh KM, Chapman KR, Decramer M, Mahler DA, Wedzicha JA. Minimal clinically important differences in pharmacological trials. Am J Respir Crit Care Med. 2014;189(3):250-255.

32. Jones PW. Estimation and application of the minimum clinically important difference in COPD. Lancet Respir Med. 2014;2(3): $167-169$.

33. Agusti A, Calverley PM, Celli B, et al. Characterisation of COPD heterogeneity in the ECLIPSE cohort. Respir Res. 2010;11:122.

34. Hanania NA, Feldman G, Zachgo W, et al. The efficacy and safety of the novel long-acting beta 2 agonist vilanterol in patients with COPD: a randomized placebo-controlled trial. Chest. 2012;142(1):119-127. 


\section{Publish your work in this journal}

The International Journal of COPD is an international, peer-reviewed journal of therapeutics and pharmacology focusing on concise rapid reporting of clinical studies and reviews in COPD. Special focus is given to the pathophysiological processes underlying the disease, intervention programs, patient focused education, and self management protocols.

This journal is indexed on PubMed Central, MedLine and CAS. The manuscript management system is completely online and includes a very quick and fair peer-review system, which is all easy to use. Visit http://www.dovepress.com/testimonials.php to read real quotes from published authors.

Submit your manuscript here: http://www.dovepress.com/international-journal-of-chronic-obstructive-pulmonary-disease-journal 